\title{
Hierarchical Formation of Dark Matter Halos near the Cutoff Scale and Their Impact on Indirect Detections
}

\author{
Tomoaki Ishiyama \\ Institute of Management and Information Technologies, Chiba University, \\ 1-33, Yayoi-cho, Inage-ku, Chiba, 263-8522, Japan \\ email:ishiyama@chiba-u.jp
}

\begin{abstract}
The smallest dark matter halos are formed first in the early universe (e.g., Hofmann et al. 2001; Berezinsky et al. 2003; Ishiyama et al. 2010). We present results of very large cosmological $\mathrm{N}$-body simulations of the hierarchical formation and evolution of halos over a wide mass range, beginning from the formation of the smallest halos. In the largest simulation, the motions of $4096^{3}$ particles in comoving boxes of side lengths $400 \mathrm{pc}$ and $200 \mathrm{pc}$ were followed. The particle masses were $3.4 \times 10^{-11} M_{\odot}$ and $4.3 \times 10^{-12} M_{\odot}$, ensuring that halos at the cutoff scale were represented by 30,000 and 230,000 particles, respectively. We found that the central density cusp is much steeper in these halos than in larger halos (dwarf-galaxy-sized to clustersized halos), and scales as $\rho \propto r^{-(1.5-1.3)}$. The cusp slope gradually becomes shallower as the halo mass increases. The slope of halos 50 times more massive than the smallest halo is approximately -1.3 . No strong correlation exists between inner slope and the collapse epoch. The cusp slope of halos above the cutoff scale seems to be reduced primarily due to major merger processes. The concentration, estimated at the present universe, is predicted to be $60-70$, consistent with theoretical models and earlier simulations, and ruling out simple power law mass-concentration relations. Such halos could still exist in the present universe with the same steep density profiles. Strongly depending on the subhalo mass function and the adopted concentration model, the steeper inner cusps of halos near the cutoff scale enhance the annihilation luminosity of a Milky Way sized halo between 12 to $67 \%$ (Ishiyama 2014).
\end{abstract}

Keywords. cosmology: theory—methods: numerical—Galaxy: structure—dark matter

\section{References}

Hofmann, S., Schwarz, D. J., \& Stöcker, H. 2001, Phys. Rev. D., 64, 083507

Berezinsky, V., Dokuchaev, V., \& Eroshenko, Y. 2003, Phys. Rev. D., 68, 103003

Ishiyama, T., Makino, J., \& Ebisuzaki, T. 2010, ApJL, 723, L195

Ishiyama, T. 2014, ApJ, 788, 27 